\title{
Cardiomiopatia Periparto. Análise Crítica da Imunossupressão
}

\author{
Heron Rached, Roberto de Cleva, Ronaldo Pinheiro, Pedro G regório M ekhitarian, Ricardo Mazzieri
}

São Paulo, SP

\begin{abstract}
É feita uma revisão da literatura a respeito da cardiomiopatia periparto, descrevendo o caso de paciente do sexofeminino, branca, 31 anos, primípara, que apresentou quadro de falência miocárdica 6 h pós-cesárea, com boa resposta após tratamento imunossupressor.
\end{abstract}

\section{Peripartum Cardiomyopathy. A critical Analysis of Immunosupression}

The authors review the literature about peripartum cardiomyopathy and describe a case of a white woman, 31 years old, primipara, who developed myocardial failure six hours after caesarian operation, with good results after immunossupressive therapy.

Arq Bras Cardiol, volume 70 (nº 4), 279-281, 1998

A cardiomiopatia periparto (CPPM) é uma doença de etiologia desconhecida, caracterizada por disfunção ventricular esquerda severa no final da gestação ou no puerpério $^{1-3}$, relatada pela primeira vez com quadro de insuficiência cardíaca idiopática com início após o parto, por Ritchie, em 1849. Uma vez que algumas pacientes desenvolvem a enfermidade no último mês da gestação, o termo atualmente empregado, miocardiopatia periparto, parece mais adequado. Os fatores de risco incluem história familiar, idade materna avançada, hipertensão arterial, deficiência de selênio, multiparidade, descendência africana e tocólise prolongada ${ }^{3-7}$. O diagnóstico em geral baseia-se em: 1) desenvolvimento de insuficiência cardíaca no último trimestre da gestação ou até seis meses pós-parto; 2) exclusão de outras causas de insuficiência cardíaca congestiva, como infecções ou toxinas e 3) ausência de cardiopatia prévia (congênita ou adquirida $)^{1}$. O tratamento imunossupressor, baseado em possível etiologia auto-imune, tem sido objeto de estudos. Relatamos o caso de uma jovem portadora de CPPM que evoluiu com recuperação plena da função miocárdica após pulsoterapia com metilpredinisolona.

\section{Relato do Caso}

Mulher de 31 anos, branca, primigesta, procedente de São Paulo, tabagista importante (20 cigarros/dia/11anos),

Hospital da Beneficência Portuguesa - São Paulo

Correspondência: Heron Rached - Unidade Coronária - Rua Maestro Cardim, 769 - 01323-001 - São Paulo, SP

Recebido para publicação em 5/1/98

Aceito em 20/1/98 pré-natal sem intercorrências, sem cardiopatia prévia ou uso de drogas, submetida a parto cesárea. Seis horas após o procedimento cirúrgico, evoluiu com queixas de dispnéia e mal estar geral. A avaliação obstétrica inicial não detectou alterações significativas. Após $24 \mathrm{~h}$, a paciente apresentou piora do quadro de dispnéia, sudorese fria e cianose de extremidades. Feito diagnóstico de insuficiência respiratória aguda, optou-se pela transferência para uma Unidade de Terapia Intensiva (UTI).

O exame físico na admissão à UTI mostrou paciente em grave estado geral, agitada, dispnéica (++++/+4), sudoréica, com palidez cutâneo-mucosa, cianose de extremidades e jugulares túrgidas $(++/+4)$ a $45^{\circ}$. A pressão arterial era 80/ $50 \mathrm{mmHg}$ e a frequiência cardíaca $140 \mathrm{bpm}$. O exame físico revelou bulhas cardíacas abafadas, com ritmo de galope (B3) e à ausculta pulmonar estertores crepitantes nos $2 / 3$ inferiores de ambos hemitóraces. A radiografia de tórax realizada à beira do leito mostrou congestão pulmonar e índice cardiotorácico de 0,6. A ecocardiografia revelou comprometimento miocárdico difuso de grau importante e discreta regurgitação valvar mitral (eco 1/fig. 1). Feito diagnóstico de choque cardiogênico, foi indicada monitorização hemodinâmica invasiva com cateter de artéria pulmonar. As variáveis hemodinâmicas confirmaram o diagnóstico de choque cardiogênico (SG 1/fig. 2). Orientados pelo quadro clínico e parâmetros hemodinâmicos, optou-se por tratamento convencional com digital, diuréticos e drogas vasoativas. Foi instituída ventilação mecânica modo assistida-controlada. Após 24h de tratamento guiado pela monitorização invasiva, a disfunção miocárdica mostrou-se refratária ao tratamento (SG 2/fig. 2). Após 48h da admissão na UTI a paciente evoluiu com quadro súbito de bradicardia, hipotensão e 


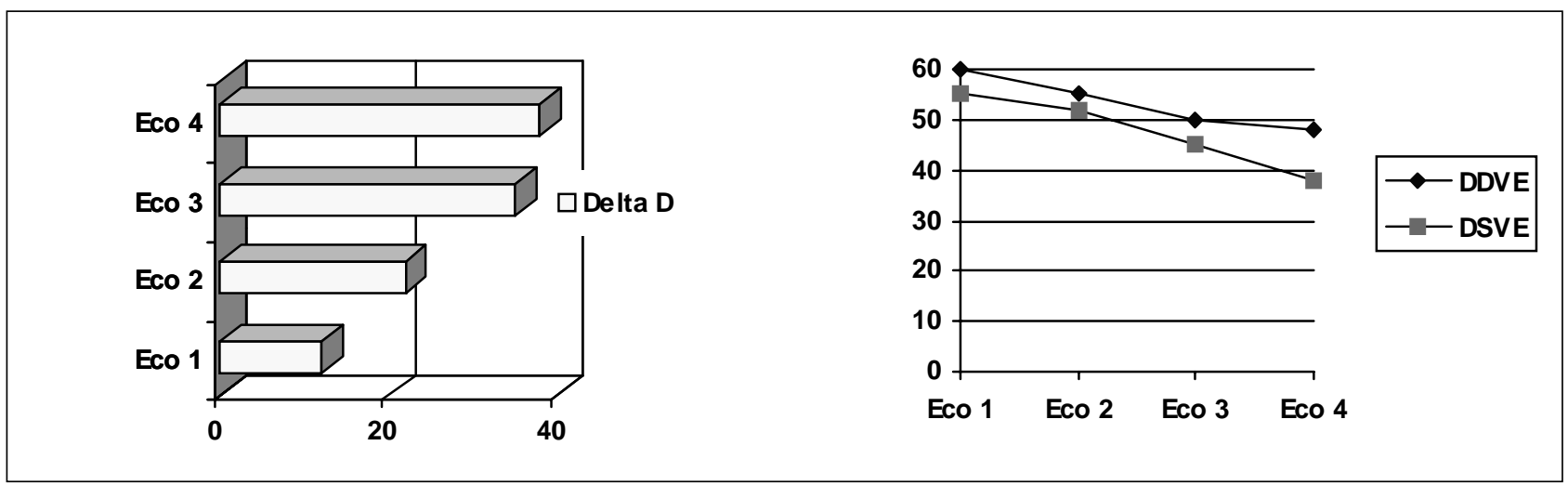

Fig. 1 - Ecocardiogramas seriados realizados à admissão (ECO1) e no $2^{\circ}(\mathrm{ECO} 2), 14^{\circ}(\mathrm{ECO} 3)$ e $28^{\circ}$ dia (ECO4) após tratamento imunossupressor. DDVE- diâmetro diastólico do ventrículo esquerdo (medidas em mm); DSVE- diâmetro sistólico do ventrículo esquerdo (medidas em mm); DELTA D- fração de encurtamento (medidas em variação percentual).

assistolia, sendo ressuscitada com sucesso. Uma hora após, as variáveis hemodinâmicas encontravam-se inalteradas em relação ao SG2. Face à gravidade do caso e refratariedade à terapêutica, resolvemos submeter a paciente à pulsoterapia com metilpredinisolona ( $1 \mathrm{~g} / \mathrm{dia} / 3 \mathrm{dias})$. Nas $48 \mathrm{~h}$ pós-tratamento imunossupressor, observamos importante melhora das variáveis hemodinâmicas (SG 3/fig. 2), com estabilização (SG 4/fig. 2) e importante melhora do desempenho ventricular esquerdo, havendo redução do diâmetro das câmaras cardíacas (eco-2/fig. 1). Realizou-se seguimento ecocardiográfico seriado no $14^{\circ}$ e $28^{\circ}$ dia pós-tratamento imunossupressor, observando-se normalização da função miocárdica e dos diâmetros cavitários (eco3/eco4/ fig. 1). A pesquisa de imunocomplexos circulantes e anticorpo anti-músculo cardíaco mostrou-se negativa.

\section{Discussão}

A CCPM é definida no contexto fisiopatológico, como miocardiopatia de padrão dilatado-congestivo, presente no último trimestre da gravidez ou, mais freqüentemente, nos seis primeiros meses pós-parto, em mulheres previamente

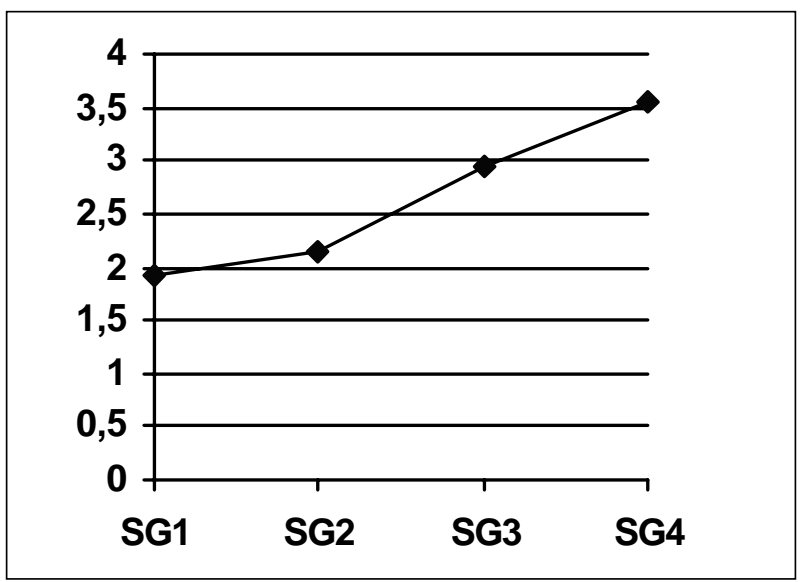

Fig. 2 - Evolução do índice cardíaco obtido com cateter de artéria pulmonar à admissão na UTI (SG1), 24h após tratamento convencional (SG2), 24h (SG3) e 48h (SG4) após tratamento imunossupressor. saudáveis ${ }^{1}$. Embora tenha sido descrita no século XIX, ainda hoje permanece com etiologia indefinida ${ }^{8}$. É considerada doença de baixa prevalência em nosso meio (1:15.000 gestantes) ${ }^{9}$ mas muito freqüente no continente africano $(1: 1.000)^{10,11}$. A CCPM é mais freqüente em pacientes de cor negra, em gestantes de idade avançada ( $>35$ a) na gestação gemelar, em gestantes com alto consumo de sódio e baixo nível sócio-econômico ${ }^{1}$.

As características hemodinâmicas, classicamente, descritas incluem aumento das pressões de enchimento com diminuição acentuada do débito cardíaco, embora alguns autores tenham encontrado circulação normal ou hiperdinâmica em percentual variável de pacientes ${ }^{12}$.

A etiologia auto-imune permanece controversa, embora já tenham sido identificados anticorpos anti-miocárdicos no sangue do cordão umbilical, anticorpos anti-actina e anti-músculo liso em portadores de CPPM ${ }^{13}$. A teoria autoimune pressupõe liberação de actomiosinas e seus metabólitos pelo útero, havendo formação de anticorpos que apresentariam reação cruzada com o miocárdio materno, causando miocardite. Entretanto, Cnac e col ${ }^{14}$ (através de dosagens séricas de IgG, IgA, IgM, complexos imunes circulantes e anticorpos anti-músculo cardíaco) e Rizeq e col ${ }^{3}$ (baixa incidência de miocardite na biópsia endomiocárdica em 34 pacientes) não conseguiram demonstrar a etiologia auto-imune.

O diagnóstico baseia-se na presença de sinais e sintomas de insuficiência cardíaca em gestante no último trimestre da gravidez ou nos seis primeiros meses pós-parto, sem doença cardíaca prévia, na ausência de abuso de drogas (álcool, cocaína ou outros depressores miocárdicos), devendo ser afastados diabetes gestacional, eclâmpsia ou pré-eclâmpsia, endocardite, tireotoxicose e edema pulmonar não cardiogênico ${ }^{1,15}$. Entretanto, deve-se ressaltar que o diagnóstico de miocardiopatia periparto pode ser feita mesmo na presença de outra cardiopatia, que justifique o quadro de insuficiência cardíaca, sendo necessária a realização de biópsia endomiocárdica ${ }^{16}$. A radiografia de tórax tipicamente revela aumento da área cardíaca com padrão congestivo pulmonar. O eletrocardiograma pode mostrar arritmias, alterações difusas da repolarização ventricular, 
bloqueio de ramo ou sinais de sobrecarga ventricular esquerda. A ecocardiografia é peça fundamental para o diagnóstico da disfunção miocárdica, além de permitir avaliar a resposta terapêutica através da análise do delta $\mathrm{D}$, fração de ejeção ventriculare diâmetros cavitários, embora tais alterações não sejam específicas. A biópsia endomiocárdica pode mostrar miocardite linfocitária ${ }^{6,8}$, infiltrado linfo-histiocitário sem necrose celular ou hipertrofia isolada de fibras cardíacas ${ }^{17,18}$. Quando a biópsia endomiocárdica é normal, a cintilografia miocárdica com índio-111 pode demonstrar a presença de miocardite ${ }^{19}$ Não foram detectados até o presente momento agentes infecciosos em associação com CPPM.

O prognóstico está diretamente relacionado ao grau de disfunção miocárdica, diâmetro das câmaras cardíacas ( diâmetro diastólico do ventrículo esquerdo >70mm) e complicações secundárias como arritmias, tromboembolismo, sepse, disfunção de múltiplos órgãos, e resposta terapêutica satisfatória (redução dos diâmetros das cavidades cardíaca e melhora no desempenho ventricular) a curto prazo (aproximadamente seis meses) ${ }^{20-22}$. A dilatação e diminuição da contratilidade miocárdica devem-se, em parte, à formação excessiva de óxido nítrico ${ }^{23}$.

Embora de eficácia duvidosa, uma vez que a etiologia auto-imune não foi adequadamente documentada, o trata- mento imunossupressor poderá ser considerado quando o paciente mostrar-se refratário ao tratamento convencional (drogas inotrópicas, diuréticos e vasodilatadores). Considerando a existência de alta incidência de cura espontânea $(50 \%)^{24}$, as complicações e efeitos colaterais dos agentes imunosupressores, seu emprego pode ser considerado medida empírica em pacientes com rápida deterioração hemodinâmica e evolução desfavorável, como no nosso caso. Entre os agentes imunossupressores podem ser empregados os costicosteróides, azatioprina ou ciclosporina. Em casos refratários, deve-se considerar a indicação de transplante cardíaco ${ }^{24,25}$.

Nosso relato descreve o caso de uma paciente na qual o emprego de imunosupressores possa ter sido benéfico, contribuindo para melhora clínica e hemodinâmica significativa após sua introdução. Observou-se melhora da função ventricular esquerda avaliada pela ecocardiografia, havendo normalização da fração de ejeção, encurtamento sistólico e diâmetros ventriculares em período de duas semanas.

Pela evolução satisfatória do caso e baseados em alguns relatos da literatura ${ }^{3,26-30}$, acreditamos que o uso criterioso de drogas imunosupressoras pode representar opção terapêutica para reverter, parcial ou totalmente, enfermidade com elevada morbi-mortalidade, ou a evolução insidiosa para cronicidade.

\section{Referências}

1. Demakis JG, Rahimtoola SH - Peripartum cardiomyopathy. Circulation 1971; 44: 964-8.

2. Lampert MB, Lang RM-Peripartum cardiomyopathy. Am Heart J 1995; 130: 86070.

3. Rizeq MN, Rickenbacher PR, Fowler MB, Billingham ME - Incidence of myocarditis in peripartum cardiomyopathy. Am J Cardiol 1994; 74: 474-7.

4. Pearl W-Familial occurrence of peripartum cardiomyopathy. Am Heart J 1995; 129:421-422.

5. Lampert MB, Hibbard J, Weinert L, Briller J, Lindheimer M, Lang RM Peripartum heart failure associated with prolonged tocolytic therapy. Am J Obstet Gynecol 1993; 168: 493-5.

6. Cnac A, Simonoff M, Moretto P, Djibo A - A low plasma selenium is a risk factor for peripartum cardiomyopathy. A comparative study in Sahelian Africa. Int J Cardiol 1992; 36: 57-9.

7. Hagley MT, Mankad SVV - Peripartum cardiomyopathy. J Am Med Wom Assoc 1991;46: 160-3.

8. Homans DC - Peripartum Cardiomyopathy. N Engl J Med 1985; 312: 1432-7.

9. Melvin KR, Richardson PJ, Olsen EGL, Daly K, Jackson G - Peripartum cardiomyopathy due to myocarditis. N Engl J Med 1982; 307: 731-4.

10. Desai D, Moodley J, Naidoo D - Peripartum cardiomyopathy: experiences at King Edward VIII Hospital, Durban, South Africa and a review of the literature. Trop Doct 1995; 25: 118-23.

11. Cnac A, Djibo A, Djangnikpo L - Peripartum dilated cardiomyopathy. A model of multifactor disease?. Rev Med Interne 1993; 14: 1033

12. Marin-Neto JA, Maciel BC, Gallo Jr. L, Almeida Filho OC, Amorim DS - Estudo hemodinâmico na cardiopatia periparto após compensação da insuficiência cardíaca. Arq Bras Cardiol 1990; 55: 93-8.

13. Rand RJ, Jenkins DM, Scott DG - Maternal cardiomyopathy of pregnancy causing stillbirth. B J Obstet Gynaecol 1975; 82: 172.

14. Cnac A, Beaufils H, Soumana I, Vetter JM, Devillechabrolle A, Moulias R-Absence of humoral autoimmunity in peripartum cardiomyopathy. Int J Cardiol 1990; 26: 49-52.

15. Leonard RB, Schwartz E, Allen DA, Alson RL - Peripartum cardiomyopathy: a case report. J Emerg Med 1992; 10: 157-61.

16. Purcell IF, Williams DO - Peripartum cardiomyopathy complicating severe aortic stenosis. Int J Cardiol 1995; 52: 163-65.
17. Sanderson EJ, Olsen EGJ, Gatei D-Peripartum heart disease: an endomiocardial biopsy study. Br Heart J 1986; 56: 285-91.

18. O' Connell JB, Costanzo-Nordin MR, Subramanian R, Robinson JA, Wallis DDE, Scanlon PJ - Peripartum cardiomyopathy: clinical, hemodynamic, histologic and prognostic characteristics. J Am Coll Cardiol 1986; 8: 52-6.

19. Navarro Gonz JF, Reviriego JLC, Mena MJ - Peripartum cardiomyopathy with normal endomyocardial biopsy and positive antimyosin-In 111 study for myocarditis. An Med Interna 1992; 9: 129-30.

20. Julian DG, Szekely P- Peripartum cardiomyopathy. Prog Cardiovasc Dis 1985; 27: 223-8.

21. Bassaw B, Ariyanayagam DC, Roopnarinesingh S-Peripartum cardiomyopathy and arterial embolism. West Indian Med J 1992; 41: 79-80.

22. Hsieh CC, Chiang CW, Hsieh TT, Soong YK - Peripartum cardiomyopathy. Jpn Heart J 1992; 33: 343-9.

23. de Belder AJ, Radomski MW, Why HJ, Richardson PJ, Martin JF - Myocardial calcium-independent nitric oxide synthase activity is present in dilated cardiomyopathy, myocarditis, and postpartum cardiomyopathy but not in ischaemic or valvar heart disease. Br Heart J 1995; 74: 426-30.

24. CettaF, Michels VV-The natural history and spectrum of idiopathic dilated cardiomyopathy, including HIV and peripartum cardiomyopathy. Curr Opin Cardiol 1995; 10: 332-8.

25. Rickenbacher PR, Rizeq MN, Hunt SA, Billingham ME, Fowler MB - Long-term outcome after heart transplantation for peripartum cardiomyopathy. Am Heart J 1994; 127: 1318-23

26. João SR, Martins JrL, Almeida Jr BN, Mariello JR, Oliveira RPS - Cardiomiopatia periparto refratária ao tratamento convencional, mas com boa resposta à medicação imunossupressora. Apresentação de um Caso. Arq Bras Cardiol 1987; 48: 49-52.

27. Midei MG, DeMent SH, Feldman AM, Hutchins GM, Baughman KL-Peripartum myocarditis and cardiomyopathy. Circulation 1990; 81: 922-8.

28. Macieira-Coelho E, Brito D, Madeirra $\mathrm{H}$ - Immunosupression therapy in peripartum myocardiopathy. Acta Med Port 1990; 3: 34-8.

29. Sardilli MHMD, Silveira LC, Lesse CR - Cardiomiopatia periparto e gravidez. Rev Soc Cardiol Est SP 1994; 6: 560-3.

30. Baughman KL, Heskowitz A, Feldman AM, Hutchins GM - Peripartum cardiomyopathy with myocarditis: who to treat. Circulation 1989; 80(suppl 1I): 320. 